\title{
Mobile and E-Healthcare: Recent Trends and Future Directions
}

\author{
Rahul K. Kher
}

Electronics and Communication Engineering Department, G H Patel College of Engineering, Vallabh Vidyanagar, India

Correcponding author: Rahul K. Kher, Electronics and Communication Engineering Department, G H Patel College of Engineering, Vallabh Vidyanagar, India, Tel: 02692231 651; E-mail: rahul2777@gmail.com

Received date: June13, 2016, Accepted date: July 01, 2016, Published date: July 10, 2016

Copyright: (C) 2016 Rahul K. Kher. This is an open-access article distributed under the terms of the Creative Commons Attribution License, which permits unrestricted use, distribution, and reproduction in any medium, provided the original author and source are credited.

Citation: Kher RK. Mobile and E-Healthcare: Recent Trends and Future Directions. J Health Med Econ. 2016, 2:10

\section{Abstract}

In the past decade, wearable sensors and devices have evolved as key technological objects which have dramatically revolutionized the next generation healthcare solutions. This is the era of cut-throat competition filled with immense stress which leads to the detection of various diseases even in the people of early age. Moreover, people are becoming more health conscious in developed as well as developing countries. The miniaturization of sensors and devices and tendency of people to be physician-independent have lead the researchers across the globe to come up with numerous healthcare solutions which are not only ubiquitous but are affordable also. Wearable medical devices (WMDs) capable of monitoring some of the most common physiological parameters- pulse, blood oxygen saturation, 2 lead ECG, heart rhythm, skin temperature etc. have already been popular. Ambulatory and long-term health monitoring for elderly people is another aspect, particularly for countries like China and Japan where population aging is increasing. With the development of networks, collected health information can be sent to the cloud server of the nearest clinic or hospital. Doctors can then provide patients with medical advice. Thus, WMDs or WMD-based applications can help both patients and doctors in daily health monitoring. The paradigm has gradually shifted to the mobile health (mHealth) - an integration of ever advancing wireless communication, ubiquitous computing, and wearable device technologies. The mHealth can be regarded as the most advanced version of healthcare monitoring.

Keywords: e-Healthcare; Healthcare solutions; Wearable; Medical devices

\section{Introduction}

The extensive usage of mobile technologies and their userfriendly applications related to health nowadays have given a rise to a new healthcare paradigm of eHealth, known as mHealth. According to the International Telecommunication
Union there are now more than 5 billion mobile phone subscriptions in the world, with over $85 \%$ of the world's population now covered by a commercial wireless signal. The growing sophistication of these networks - offering higher and higher speeds of data transmission alongside cheaper and more powerful handsets - are transforming the way health services and information are accessed, delivered, and managed. With increased accessibility comes the possibility of greater personalization and citizen-focused public health and medical care [1]. The e-healthcare and now its mobile version, m-healthcare, system basically include wireless body sensor networks (WBSN) or/and wireless personal area networks (WPAN) for providing higher-quality medical services and more efficient medical responses and treatments to patients. In such an e-healthcare/ $\mathrm{m}$-healthcare systems, the sensors placed around patients' body gather the vital parameters (e.g. heart rate, pulse rate, oxygen saturation level, sugar level and many more) andreport them to remote healthcare service provider and/ or physician immediately for real-time and long-term monitoring of patients [2]

The details of earlier days e-healthcare systems are found in [3-8]. The mobile and/or smart-phone based health motoring systems have been reported in [9-14]. The e-healthcare systems have come up with models and formats viz. tele-home healthcare comprising of cuffless blood pressure meter, ringtype heart rate monitor and Bluetooth-based ECG monitor [15], wrist-wearable telemedicine monitor for heart patients (AMON) and a generic belt-integrated computing platform for home and hospital use (QBIC) [16], physiological parameter monitoring through mobile/smart phone [17] and many more reported in [18-25].

There are many aspects as well as challenges associated with the mobile and e-healthcare systems:

- Architectural design and implementation issues

- Integration of data and networks

- Secure and efficient data transmission and management

- Maintaining privacy of patient information and their data

This paper discusses the current status of mobile and ehealthcare systems in context of above aspects and challenges as well as upcoming, future trends. Section 2 describes various e-healthcare models and systems implemented till now along 
with upcoming technologies; section 3 presents the application domains of mobile and e-healthcare; section 4 briefs the challenges and security issues encountered in mobile and e-healthcare systems and section 5 briefly provides the insight to the future scopes in mobile/e-healthcare.

\section{Various e-healthcare models and systems}

\section{Wireless Body Sensor Network (WBSN) based e-healthcare Systems:}

There are many reasons for innovating the mobile and ehealthcare systems e.g. a daily monitoring of vital physiological parameters to the detection of an abnormal event accomplished by ambulatory monitoring. A tele-home healthcare system [15], for example, has been proposed for remote patient monitoring. The system utilizes wearable devices, wireless communication technologies, and multisensory (cuffless blood pressure meter, ECG and PPG sensors) data fusion methods. In [26], authors have proposed a body sensor network (BSN) which allocates extra resources for important parameter like ECG maintaining the security of data over the network. The integrated network of IEEE 802.11/ WLAN and IEEE 802.16/WiMAX can bring a synergetic improvement to the telemedicine services on coverage, data rates and quality of service (QoS) provisioning to mobile users. This integrated network will be able to provide services like emergency telemedicine, mobile medical data, mobile robotic system and pre-hospital care [27]. The healthcare and patient monitoring systems based on 2G-RFID, Bluetooth-enabled inhome patient monitoring system and multitier wireless network using 802.15.4 low data rate WPAN with 802.11 WLAN have been proposed in [28-30].

Smart wearable garments like ProeTEX (Protective Electronic TEXtiles for emergency operators) [14] and Heally recording system, having ECG/EMG/EOG electrodes, various electronics modules and $\mathrm{NiMH}$ battery) mounted on a shirt [8]. In a sensorized T-shirt is developed automatic sleep classificationwake, rapid eye movement (REM) and non REM- based on heart rate variability (HRV), respiration and movement signals. Wirelessly powered fabric patch sensor system for ECG recording at very low power has been developed in.

\section{Cellular and smartphone based e-healthcare systems:}

A Mobicare cardio monitoring system consisting of a cellular phone embedded with real time ECG processing algorithmsdetection of QRS complex, $\mathrm{Q}$ onset, $\mathrm{T}$ offset- (MobiECG), a bluetooth-enabled ECG sensor, a web-based server, a patient's database and a user interface is presented in [6]. In this framework, ECG processing is carried out by MobiECG and it will send the abnormal ECG data over a cellular network (GPRS/3G) to hospitals or care centres to alarm physician only when it detects abnormal ECG signal. Otto et al. [31] have presented a WBAN and TinyOS based architecture for monitoring body motion and heart activity. The system consists of a network coordinator and a personal server running on a personal digital assistant (PDA). HeartToGo- a windows mobile smartphone-based wearable cardiovascular diseases (CVD) detection system; capable of performing realtime ECG acquisition and display, feature extraction, and beat classification has been developed by Oresko et al. [9]. The system is capable of classifying the premature ventricular contraction (PVC) as well as generating the cardiac summary report consisting of the average, high, and low heart rate, and the total number of beats, as well as the number of normal and PVC beats.

Cellular phone based patient monitoring system (C-SMART) [32], mobile phone based fall detection system on Android platform [33], personal heart monitoring and rehabilitation system using smart phones [34], a mobile health monitoring system for elderly people (iCare) [35], MobiHealth- a vital telemonitoring and tele-treatment system based on a body area network (BAN) and mobile healthcare service platform using next generation public wireless networks [36], smartphone based body area network system (SBBANS) [37], Android based fall detection system with physiological data monitoring [38], mobile based multi-function health monitoring system [39], human activity recognition system combining accelerometer data and GPS of android- based smartphone [40], daily mood assessment using mobile phone sensing [41], a mobile EOG-based human computer interface for assistive healthcare (EyePhone) [42], smartphone-based sleep quantity measuring and modelling algorithm [43], an android-based mobile healthcare system incorporating PSoC and cloud storage [44], smartphone-based continuous monitoring system for home-bound elders and patients [45] have been found in literature which have extensively used cellular phones and smartphones for efficient, wearable and portable healthcare systems.

In addition to above mentioned systems, a portable, lightweight wearable healthcare monitoring systems have been proposed in $[4,5,7,46]$.

\section{System on Chip (SoC) e-healthcare modules:}

A portable system on chip for ECG monitoring has been developed [47] which is capable of implementing configurable functionality with low-power consumption. The SoC is implemented in $0.18 \mu \mathrm{m}$ CMOS process and consumes $32 \mu \mathrm{W}$ from a $1.2 \mathrm{~V}$ while heart beat detection application is running, and integrated in a wireless ECG monitoring system with Bluetooth protocol. A low-power biosignal acquisition and classification system consisting of: 1) a high-pass sigma delta modulator based biosignal processor (BSP) for signal acquisition and digitization, 2) a low-power, superregenerative on-off keying transceiver for short-range wireless transmission, and 3) a digital signal processor (DSP) for ECG classification is proposed in [48]. In this system too, the chips are fabricated in TSMC $0.18 \mu \mathrm{m}$ standard CMOS process. An airflow sensing chip integrating the MEMS sensors with their CMOS signal processing circuits is proposed for respiration detection. The airflow sensors have been fabricated using a $0.35 \mu \mathrm{m}$ CMOS/MEMS 2P4M mixed signal process [49].

In [50], a wearable neuro-feedback system is proposed with a low-power neuro-feedback SoC (NFS) for mental status monitoring with EEG and transcranial electrical stimulation 
(tES). An embedded chip that supports independent component analysis (ICA) and support vector machine (SVM) is fabricated in $130 \mathrm{~nm}$ CMOS technology. Vaidya et al. [44] have developed a system that measures the ECG, blood pressure and heart rate of the patient. After acquiring and filtering the ECG and PPG signals, they are fed to Cypress Programmable System on Chip 3 (PSoC 3 ) for digitizing them and calculating the heart rate and blood pressure. The capability of mixed signal processing of PSoC 3 adds the compactness and portability to the system. A few more SoC designs for lowpower healthcare systems are listed in [51-53].

\section{Cloud-based e-healthcare systems:}

With the evolvement of mobile and e-healthcare technologies, the concept of health-cloud has emerged. In [54], patient's health monitoring using Health-Cloud has been presented. When the location of a mobile patient changes, the associated default gateway also changes and, consequently, the optimum mapping between the server and the mobile node also changes. An auction theory based optimal resource allocation framework for health-cloud to monitor patients' health condition is proposed in [54]. Existing cloud-based ehealthcare applications provide accesses to their services using single-sign-on (SSO) protocols. Authors in [55] have proposed a scheme called, chord for cloud (CoC), to overcome the increased execution load on cloud gateway as well as identity provider. Other issues related with the cloud-based ehealthcare systems like data security; compression of data/ images; a combined cryptographic and watermarking techniques for combating the insider attacks on patients' medical data; enhancing computational capacity, particularly for multimedia medical data; database management systems for accessing the sensitive data via private cloud and public data via public cloud, thus forming a hybrid cloud; privacypreserving protocol for dynamic medical text mining (PPDM) in cloud assisted e-healthcare system have been addressed in [56-60].

\section{Application Domains of Mobile and E- Healthcare}

The e-healthcare systems have primarily been developed for observing the physiological parameters like body temperature, oxygen saturation level, heart rate, blood glucose level etc. However, with evolution of wireless technologies there has been a manifold variation and enhancement in the $\mathrm{e}$ healthcare systems. The WBSN based multi-parameter acquisition systems have come up for various applications ranging from ambulatory monitoring to a high-end research.

\section{Tele-Home patient monitoring}

It has been observed that, primarily, the mobile and ehealthcare systems have been designed for home-based or remote patient monitoring. Tele-Home patient monitoring is one of the most significant and prevailing applications of mobile and e-healthcare. The majority tele-home patient monitoring systems include recording and observing the cardiac parameters $[3-10,13,26,47,48,60]$. However, gradually the range of sensors/ data and applications got widen to include other vital physiological parameters (blood pressure, oxygen saturation level in blood $\left(\mathrm{SpO}_{2}\right)$, pulseplethysmogram (PPG) signal, blood glucose level, respiration rate) $[4,7,11,12,15-18,36,37]$, EEG/ EOG signals [20,42,50], body movements (physical) activities and stress level [14,40], sleep/ wake detection [8], mood assessment [41], fall detection in elderly people [33,38], weight loss and maintenance [22] etc.

The compact, user-friendly and cost-effective tele-home patient monitoring has become possible after the smart phones with many in-built sensors and integrated technologies have been introduced. Nawka et al. [25] have proposed, SESGARH: a Scalable Extensible Smart-Phone based Mobile Gateway and Application for Remote Health monitoring. Using SESGARH, patients send disease symptoms and other critical health information to a physician, who can analyze this data remotely and prescribe e-prescriptions. SESGARH can significantly minimize the time taken for transmission of healthcare data from patient to physician and vice-versa, and improve the reach and quality of healthcare services. The proposed system is based on the ISO/IEEE11073 (X73) family of standards supporting high quality medical sensors, as well as wireless transport (e.g. Bluetooth, WiFi, Zigbee) for access to faster and reliable communication network resources.

A portable heart monitoring and cardiac rehabilitation system based on smart phone has been developed by Leijdekkers et al. [34]. The system focuses mainly on:

1. continuous heart/ECG monitoring, in which the monitoring device, in case of emergency calls the ambulance/ caregivers intimating the location of a patient.

2. Cardiac rehabilitation, which aims encouraging the patient to perform his/her physical exercises and making lifestyle changes such as getting more active, losing weight, reducing stress or quit smoking through interactive features of a smart phone. K. Wac et al. [36] have developed the MobiHealth-mobile patient monitoring system. The developed system allows the incorporation of diverse medical sensors via wireless connections, and the live transmission of the measured vital signs to healthcare providers as well as realtime feedback to the patient. Ziyu Lv et al. [35] and Megalingam et al. [45] have developed the smartphone based health monitoring systems for elderly patients. The typical features of these systems include wireless body sensors for vital parameters (heart rate, body temperature, ECG, respiration rate, tilt and fall detection) monitoring, smartphone, automatic alert/call to pre-assigned people and ambulance.

\section{Abnormalities detection}

With the increased processing power of mobile devices, it has been possible to detect the abnormalities such as arrhythmia or cardiovascular diseases (CVD) from the acquired physiological data/ signals. An early and, in some cases, real time detection of such abnormalities will be able to provide quick treatment to the patient. Fensli et al. [7] have proposed 
one of the earlier online arrhythmia monitoring systems. The scheme consists of wearable ECG recording system along with a server based arrhythmia calculating and monitoring mechanism, which will let the physician know about the arrhythmic condition, if any. The patient will get the follow up from physician through an internet enabled personal digital assistant (PDA). X Chen et al. [6] have developed cellphone based ambulatory and continuous ECG signal processing systems. The Mobicare cardio monitoring system consists of a cellular phone embedded with real time ECG processing algorithms (MobiECG), a wireless ECG sensor, a web based server, a patients' database and a user interface. The system is capable of detecting the condition of atrial fibrillation (AF) and display the maximum heart rate $(\mathrm{MH})$ and intensity of patient's body movements on GUI.

A real time CVD detection based on smartphone platform has been developed by Oresko et al. [9]. HeartToGo- an experimental prototype consisting of Alive ECG sensor and Windows smartphone has been used for detecting the premature ventricular contraction (PVC) beats. In the process, the LabVIEW mobile module for QRS detection and artificial neural network (ANN) based PVC beat classification have been accurately implemented. Lin et al. [60] have proposed an intelligent telecardiology system using a wearable and wireless ECG to detect atrial fibrillation. A Java based mobile system software for QRS detection algorithm, sinus tachycardia/ bradycardia based on heart rate and two algorithms for atrial fibrillation have been developed.

\section{Specific applications}

In this section the mobile/e-healthcare applications discussed are focused on cardiac abnormality detection. Researchers have proposed systems involving other biomedical signals like EEG, EMG, EOG etc. for some specific applications. Accurate estimation of shifts in drivers' levels of arousal, fatigue and vigilance for safe driving has drawn attention in recent times. Chin-Teng Lin et al. [20] have developed a wireless and wearable EEG system for evaluating driver vigilance. The proposed system- called Mindo, monitors in real time, the fluctuations in driving performance with changes in brain activities. The system, implemented using JAVA as mobile application for online analysis, processes the EEG recording to translate them into the vigilance level of drivers. A wearable neuro-feedback system with EEG and transcranial electrical stimulation (tES) based mental status monitoring has been developed by Roh et al. [50]. The proposed system incorporates neuro-feedback SoC architecture for implementing independent component analysis (ICA), FFT and support vector machine (SVM).

Erik English et al. [42] have proposed a mobile EOG based $\mathrm{HCl}$ (EyePhone). The prototype, still at early stage, allows users to control mobile phones through intentional eye or facial movements. The graphical window can receive and display continuous EEG data acquired from the headset; a mouse emulator can allow users to move a cursor around the smartphone screen by moving their heads and eyes; and an emergency dialler can allow users to make an emergency phone call through a certain pattern of eye/facial movements.

Recovering the hand functionality lost or reduced by injuries, or chronic diseases requires a kinesiotherapic intervention in addition to beyond pharmacological treatments [61-65]. Pani et al. [66] developed a novel device with remote monitoring capabilities for the needs of rheumatic patients. It comprises several sensorized tools and can be used either in an outpatient clinic for hand functional evaluation, connected to a PC, or given to the patient for home kinesiotherapic sessions. In the latter case, the device guides the patient in the rehabilitation session, transmitting the relevant statistics about his performance to a TCP/IP server exploiting a GSM/GPRS connection for later analysis.

Other specific applications of mobile/ e-healthcare based systems include smartphone based wound image analysis system for detecting foot ulcer in diabetic patients [21]; myPace: an integrative health platform for supporting weight loss and healthy eating behaviours [22]; MoodMiner- human behaviour pattern and daily mood assessment system using smartphone sensor and communication data (acceleration, light, location call log etc.) [41]; android smartphone based fall detection application which includes collection of accelerometer data, learning the fall behaviour pattern with collected data and alerting the caregivers upon fall detection $[33,38]$.

\section{Challenges and Security Issues in e- Healthcare Systems}

\section{Challenges with WMDs and e-healthcare}

The e-healthcare systems deal with patient-centric critical medical information, where accuracy of data is of prime concern. Following major challenges are imposed in ehealthcare systems.

Usability: Despite non-trivial efforts from $\mathrm{CHI}$ (ComputerHuman Interaction) research domain, the unique requirements on the design of user-friendly e-Health monitoring systems raise a set of challenges. Lack of friendly interfaces/GUI for both medical staff and patients is one of the major factors impeding their wider acceptance in practice. In addition, as patient-centric monitoring relies on the cooperation of multiple heterogeneous sensors, ranging from wearable sensors to smartphone and IOT sensors, their configurations must be transparent to the patients, avoiding any confusing or inconvenient settings [67].

Sensor data/ information quality and Cost-effectiveness: Quality of the sensor data/ information collected must be ensured, as the noisy or false data will lead to incorrect action or diagnosis. This will impose a trade-off between the cost of the system and quality of data. The sensors may collect and report false or noisy data due to unexpected hardware failures or unreliable communications issues which will lead false interpretation and analysis of the data. Further, due to several WBANs located in small area, particularly in the hospitals, 
there could be inter sensor interference or electromagnetic interference with sensor data [67].

One of the major challenges and concerns associated with WMDs and e-healthcare systems is the affordability and costeffectiveness. These facilities should be made available to the low-income and middle-class people as well. In last five years, the cost of electronics and computational hardware has dramatically reduced.

This should be a positive sign for providing the cost-effective e-healthcare facilities to the lower or poor people.

Additionally, several other issues like sensor node failure/ removal, attack on BAN/WBAN from external attacker, environmental interference like signal fading, limited or loss of power/ battery, loss of connectivity/ network failure due to transceiver failure or network interruption, network congestion due to excessive sensor data, and most importantly, compatibility/ interoperability due to different network standards $[68,69]$ should be carefully addressed while designing the e-healthcare systems.

\section{Privacy and security of patients' data and information}

To guarantee the security and privacy of patients physiological data and information is one of the major issues in WBANs. The data security and privacy issues in WBANs have first been investigated comprehensively by Ming Li et al. [70]. They have looked into two important data security issues: secure and dependable distributed data storage, and finegrained distributed data access control for sensitive and private patient medical data. Lim S [71] reports various security attacks and weaknesses in the wireless topologies like Bluetooth and GSM. For example, vulnerabilities in Bluetooth securities arise due to various attacks like MAC spoofing attack, (2) PIN cracking attack (3) Man in the Middle Attack, (4) Blue Jacking Attack, (5) Blue Snarfing Attack, (6) Blue Bugging Attack, (7) Blue Printing Attack, (8) Blue over Attack, (9) Off line PIN recovery Attack, (10) Brute Force Attack, (11) Reflection Attack, (12) Backdoor Attack, (13) DOS Attack, (14) Cabir Attack, (15) Skulls Worm Attack, and (16) Lasco Worm Attack. Similarly, GSM and over the air voice security and privacy are affected by weaknesses in streaming ciphers like $A 5 / 1$ and $A 5 / 2$.

Jun Zhou et al. [57] have proposed a privacy-preserving protocol for dynamic text mining (PPDM) for cloud assisted ehealthcare systems. Based on the proposed protocol, PPDM1privacy preserving function correlation matching from dynamic medical text mining and PPDM2- privacy preserving medical image feature extraction design have been implemented. The mobility based healthcare systems/ devices allow patients to move easily. Thus, when patients move their topology and path change and in order to stay connected securely, for patients, a secure session-key is highly required. Pardeep Kumar et al. [69] have addressed a secure session-key scheme for mobility supported e-healthcare systems. In the proposed scheme, authors have considered replay attack, man-in-themiddle (MITM) attack and impersonation attack for security analysis. The performance analysis of the scheme has been made using the parameters- communication cost and computation cost. Song et al. [53] have combined the orthogonal matching pursuit (OMP) based encryption and compressed sensing (CS) based compression for secure image storage for cloud-based e-healthcare system.

Some more security and privacy issues and concerned solutions in wireless and remote patient monitoring systems are reported in [71-73].

\section{Future Directions}

A strategic study at the National Institutes of Health (NIH) has noted: "With the development of miniaturized devices and wireless communication, the way in which doctors care for patients will change dramatically and the role patients take in their own healthcare will increase. Healthcare will become more personalized through tailoring of interventions to individual patients" [74]. Though the challenges of providing high-quality healthcare in developing countries are different than those in developed countries, they share a common goal: to provide access to health monitoring and assessment technologies to people with limited or no healthcare facilities, or with geographically distant or difficult to physically access facilities.

The increasing number of mobile devices, ubiquitous internet and cloud computing will progressively emerge into a new domain called Wearable Internet of Things (WIOT). This will lead to increased capability of sensing, computing and communication. Future generations of WloT promise to transform the healthcare sector, wherein individuals are seamlessly tracked by wearable sensors for personalized health and wellness information. However, to achieve multidimensional success, WloT needs not only to overcome the technical challenges of generating a flexible framework for networking, computing, storing, and visualizing, but also needs to consolidate its position in designing solutions that are clinically acceptable and operational [75].

The pervasive use of smartphones and the ubiquity of WiFi connections will enable medical informatics to overcome the time and location barriers. This method should especially be useful in those instances in which a rapid response is critical or when conditions are changing dynamically. A rich set of embedded sensors (e.g., camera, accelerometer, vibrating gyroscope, magnetometer, goniometer, actometer, pedometer, pressure sensor and more) of the smartphones will enable revolution in nearly all aspects of healthcare. The unobtrusive and intelligent smartphone-based healthcare device/ system will make it more popular and cost-effective, too as no extra hardware/ technologies will be required [76]. All in all, the future of healthcare belongs to loT-centric smart healthcare systems [77] with a possible intelligent self-disease management owing the powerful mobile and smartphone technologies [78]. The assistance and effectiveness of WMDs and e-healthcare systems for the people suffering from chronic/ life threatening diseases should also be properly investigated. 


\section{Conclusion}

The recent years have shown a tremendous growth in ehealthcare thanks to the ever evolving wireless and sensor technologies. The relatively larger e-healthcare architecture has become light, convenient and cost-effective with the advent of smartphones. The mobile and e-healthcare technologies have many fold applications and spread i.e. from monitoring the vital physiological parameters-heart rate, $\mathrm{SpO} 2$, blood pressure, blood glucose level, ECG etc. - to detecting various abnormalities like tachycardia, bradycardia, stress, atrial fibrillation, seizure etc. Indeed, with the available mobile and e-healthcare systems and devices have drastically changed the nature of health management and treatment. However, there many aspects like data security/ privacy and costeffectiveness need to be addressed. Looking forward to the future of mobile/ e-healthcare systems, it is more than clear that IOT and smartphones with multi-functionality medicinal feature will bring the intelligence, ease and smartness in the global healthcare sector.

\section{References}

1. http://www.who.int/goe/publications/goe_mhealth_web.pdf

2. Shen X, Kato N, Lin X (2010) Wireless Technologies for ehealthcare. IEEE Wireless Communications. pp. 10-11.

3. Shojaei-Baghini M, Lal RK, Sharma DK (2005) A Low-Power and Compact Analog CMOS Processing Chip for Portable ECG Recorders. Asian Solid State Circuit Conference, A-SSCC'05, Hshinchu, Taiwan.

4. Okada Y, Yoto YT, Suzuki TA, Sugiura T, Sakuragawa S (2010) Development of a Wearable ECG Recorder for Measuring Daily Stress. Int Conf on Information Science and Applications (ICISA), Seoul, Korea. pp. 21-23.

5. Park C, Chou PH, Bai Y, Matthews Y, Hibbs A (2006) An UltraWearable Wireless Low Power ECG Monitoring System. Biomedical Circuits and Systems Conference, London, UK. pp: 241-244.

6. Chen X, Ho CT, Lim ET, Kyaw TZ (2007) Cellular Phone Based Online ECG Processing for Ambulatory and Continuous Detection. Computers in Cardiology, Durham, NC, USA. pp. 653656.

7. Fensli R, Gunnarson E, Gundersen T (2005) A Wearable ECGrecording System for Continuous Arrhythmia Monitoring in a Wireless Tele-Home-Care Situation. 18th IEEE Symposium on Computer based Medical Systems. pp: 407-412.

8. Karlen W, Mattiussi CL, Floreano D (2009) Sleep and Wake Classification with ECG and Respiratory Effort Signals. IEEE Transactions on Biomedical Circuits and Systems 3: 71-78.

9. Oresko JJ, Jin Z, Cheng J, Huang S, Sun Y, et al. (2010) A Wearable Smartphone-Based Platform for Real-Time Cardiovascular Disease Detection Via Electrocardiogram Processing. IEEE Transactions on Information Technology in Biomedicine.

10. Fletcher RR, Kulkarni S (2010) Clip-on Wireless Wearable Microwave Sensor for Ambulatory Cardiac Monitoring. 32nd Annual International Conference of the IEEE EMBS, Buenos Aires, Argentina.
11. Gallego J, Lemos D, Meneses GA, Hernandez AM (2010) Development of a Wearable Vital Signs Monitor for Healthcare. 32nd Annual International Conference of the IEEE EMBS, Buenos Aires, Argentina.

12. Figueiredo CP, Becher K, Hoffmann KP, Mendes PM (2010) Low Power Wireless Acquisition Module for Wearable Health Monitoring Systems. 32nd Annual International Conference of the IEEE EMBS (EMBC 2010), Buenos Aires, Argentina.

13. Hwang S, Trakimas M, Sonkusale S (2010) A Low-Power Asynchronous ECG Acquisition System in CMOS Technology. 32nd Annual International Conference of the IEEE EMBS (EMBC 2010), Buenos Aires, Argentina.

14. Curone D, Tognetti A, Secco EL, Anania G, Carbonaro N, et al. (2010) Heart Rate and Accelerometer Data Fusion for Activity Assessment of Rescuers During Emergency Interventions. IEEE Transactions on Information Technology in Biomedicine 14: 3.

15. Hung K, Zhang YT, Tai B (2004) Wearable Medical Devices for Tele-Home Healthcare. Proceedings of the 26th Annual International Conference of the IEEE EMBS, San Francisco. pp: 5384-5387.

16. Scheffler M, Hirt E (2004) Wearable devices for emerging healthcare applications. Proceedings of the 26th Annual International Conference of the IEEE EMBS, San Francisco. pp: 5384-5387.

17. Scully CG, Lee J, Meyer J, Gorbach AM, Granquist-Fraser D, et al. (2012) Physiological Parameter Monitoring from Optical Recordings with a Mobile Phone. IEEE Transactions on Biomedical Engineering 59: 303-306.

18. Seeger C, Laerhoven KV, Buchmann A (2015) My Health Assistant: An Event-driven Middleware for Multiple Medical Applications on a Smartphone-Mediated Body Sensor Network. IEEE Journal of Biomedical and Health Informatics 19: 752-760.

19. Simplicio MA, Iwaya LH, Barros BM, Tereza CMB, Carvalho, et al. (2015) SecourHealth: A Delay-Tolerant Security Framework for Mobile Health Data Collection. IEEE Journal of Biomedical and Health Informatics 19: 761-772.

20. Lin CT, Chun-Hsiang C, Chih-Sheng H, Shu-Fang T, Shao-Wei L, et al. (2014) Wireless and Wearable EEG System for Evaluating Driver Vigilance. IEEE Transactions on Biomedical Circuits and Systems 8: 165-176.

21. Wang L, Pedersen PC, Strong DM, Tulu B, Agu E, et al. (2015) Smartphone-Based Wound Assessment System for Patients with Diabetes. IEEE Transactions on Biomedical Engineering 62: 477-488.

22. Barnett J, Harricharan M, Fletcher D, Gilchrist B, Coughlan J (2015) myPace: An Integrative Health Platform for Supporting Weight Loss and Maintenance Behaviors. IEEE Journal of Biomedical and Health Informatics 19: 109-116.

23. Huang L, Xu Y, Chen $\mathrm{X}, \mathrm{Li} H, \mathrm{Wu} Y$ (2012) Design and Implementation of Location Based Mobile Health System. 4th International Conference on Computational and Information Sciences, Chongqing, China. pp: 919-922.

24. Nkosi MT, Mekuria F (2010) Cloud Computing for Enhanced Mobile Health Applications. 2nd IEEE International Conference on Cloud Computing Technology and Science, Indianapolis, USA. pp: 629-634.

25. Nawka N, Maguliri AK, Sharma D, Saluja P (2011) SESGARH: A Scalable Extensible Smart-Phone based Mobile Gateway and 
Application for Remote Health Monitoring, Bangalore, India. pp: 1-6.

26. Wang H, Peng DD, Wang W, Hamid Sharif, Hsiao- Hwa Chen (2010) Resource-Aware Secure ECG Healthcare Monitoring through Body Sensor Networks. IEEE Wireless Communication. pp:12-19.

27. Zhang $Y$, Ansari N, Hiroshi Tsunoda (2010) Wireless Telemedicine Services Over Integrated. IEEE 802.11/WLAN and IEEE 802.16/ WiMAX Networks, IEEE Wireless Communication. pp: 30-36.

28. Chen M, Gonzalez S, Leung V, Zhang Q, Li M (2010) A 2G-RFIDbased e-healthcare system. IEEE Wireless Communication. pp: 37-43.

29. Cheng TH, Zhuang W (2009) Bluetooth-enabled in-home patient monitoring system: Early detection of Alzheimer's disease. IEEE Wireless Communication. pp: 74-79.

30. Misic J, Misic VB (2010) Bridge Performance in A Multitier Wireless Network for Healthcare Monitoring. IEEE Wireless Communication. pp: 90-95.

31. Otto C, Milenković A, Sanders C, Jovanov E (2006) System Architecture of a Wireless Body Area Sensor Network for Ubiquitous Health Monitoring. Journal of Mobile Multimedia 1: 307-326.

32. Blumrosen G, Avisdris N, Kupfer R, Rubinsky B (2011) C-SMART: Efficient Seamless Cellular Phone Based Patient Monitoring System 2011 IEEE Int. Symp. on a World of Wireless, Mobile and Multimedia Networks (WoWMoM), Lucca, Italy. pp: 1-6.

33. Fang SH, Liang Y, Chiu KM (2012) Developing a Mobile Phonebased Fall Detection System on Android Platform. 2012 Computing, Communications and Applications Conference (ComComAp), Hong Kong. pp: 143-146.

34. Leijdekkers P, Gay V (20006) Personal Heart Monitoring and Rehabilitation System using Smart Phones. Int Conf on Mobile Business (ICMB '06), Copenhagen. pp: 29-35.

35. Lv Z, Xia F, Wu G, Yao L, Chen Z (2010) iCare: A Mobile Health Monitoring System for the Elderly. 2010 IEEE/ACM Int Conf on Green Computing and Communications (GreenCom) \& Int Conf on Cyber, Physical and Social Computing (CPSCom), Hangzhou, China. pp: 699-705.

36. Wac K, Bults R, Beijnum B, Widya I, Jones V, et al. (2009) Mobile Patient Monitoring: the MobiHealth System. 31st Annual Int Conf of the IEEE EMBS (EMBC 2009), Minneapolis, Minnesota, USA. pp: 1238-1241.

37. Shi Y, Zhang Y (2014) Smartphone Based Body Area Network System. 2014 International Conference on Medical Biometrics, Shenzhen, China. pp: 204-209.

38. Koshmak GA, Linden M, Loutfi A (2013) Evaluation of the Android-Based Fall Detection System with Physiological Data Monitoring. 35th Annual Int Conf of the IEEE EMBS (EMBC 2013), Osaka, Japan. pp: 1164-1168.

39. Kim BJ, Noh YH, Jeong DH (2013) Implementation of Mobile based Multi-Function Health Monitoring System. 2013 International Conference on IT Convergence and Security (ICITCS), Macao, Hong Kong. pp: 1-4.

40. Kaghyan S, Sarukhanyan H (2013) Accelerometer and GPS Sensor Combination Based System for Human Activity Recognition. 2013 Int Conf on Computer Science and Information Technologies (CSIT), Yerevan, Armenia. pp: 1-9.
41. Ma Y, Xu B, Bai Y, Sun G, Zhu R (2012) Daily Mood Assessment based on Mobile Phone Sensing. 2012 9th Int Conf on Wearable and Implantable Body Sensor Networks (BSN), London, UK. pp: 142-147.

42. English E, Hung A, Kesten E, Latulipe, Zhanpeng Jin (2013) EyePhone: A Mobile EOG-based Human-Computer Interface for Assistive Healthcare. 6th Annual Int. IEEE/EMBS Conf. on Neural Engineering (NER), San Diego, California. pp: 105-108.

43. Alvika Gautam, Vinayak S. Naik, Archie Gupta, S. K. Sharma, Sriram K (2015) An Smartphone-based Algorithm to Measure and Model Quantity of Sleep. 2015 7th Int Conf on Communication Systems and Networks (COMSNETS), Bangalore, India. pp: 1-6.

44. Avinash S. Vaidya, M.B. Srinivas, P. Himabindu, Daria Jumaxanova (2013) A Smart Phone/Tablet based Mobile Health Care System for Developing Countries", 35th Annual Int. Conf. of the IEEE EMBS (EMBC 2013), Osaka, Japan. pp: 4642-4645.

45. Rajesh Kannan Megalingam, Goutham Pocklassery, Vivek Jayakrishnan, Galla Mourya, Athul Asokan Thulasi (2014) Smartphone Based Continuous Monitoring System for Homebound Elders and Patients. 2014 International Conference on Communications and Signal Processing (ICCSP), Melmaruvathur, India. pp: 1173-1177.

46. Zheng JW, Zhang ZB, Wu TH, Zhang Y (2007) A wearable mobihealth care system supporting real-time diagnosis and alarm. Med Bio Eng Comput 45: 877-885.

47. Kim H, Kim S, Helleputte NV, Artes A, Konijnenburg M, et al. (2014) A Configurable and Low-Power Mixed Signal SoC for Portable ECG Monitoring Applications. IEEE Transactions on Biomedical Circuits and Systems 8: 257-267.

48. Shuenn-Yuh L, Jia-Hua H, Cheng-Han H, Ming-Chun L, Shih-Yu CC, et al. (2015) Low-Power Wireless ECG Acquisition and Classification System for Body Sensor Networks. IEEE Journal of Biomedical and Health Informatics 19: 236-246.

49. Wei CL, Lin YC, Chen TA, Lin TY, Hao TL (2015) Respiration Detection Chip With Integrated Temperature-Insensitive MEMS Sensors and CMOS Signal Processing Circuits. IEEE Transactions on Biomedical Circuits and Systems 9: 105-112.

50. Roh T, Song K, Cho H, Shin D, Yoo HJ (2014) A Wearable NeuroFeedback System with EEG-Based Mental Status Monitoring and Transcranial Electrical Stimulation. IEEE Transactions on Biomedical Circuits and Systems 8: 755-764.

51. Das S, Misra S, Khatua M, Rodrigues JJPC (2013) Mapping of Sensor Nodes with Servers in a Mobile Health-Cloud Environment. IEEE 15th Int. Conf. on e-Health Networking, Applications and Services (Healthcom 2013), Lisbon, Portugal. pp: 481-485.

52. Kim IK, Pervez Z, Khattak AM, Lee S (2010) Chord Based Identity Management for e-Healthcare Cloud Applications. 10th Annual International Symposium on Applications and the Internet, Seoul, Korea. pp: 391-394.

53. Song C, Lin X, Shen XS (2013) Secure and Effective Image Storage for Cloud based E-healthcare Systems. IEEE Global Communication Conference (GLOBECOM), Atlanta, USA. pp: 653-658.

54. Garkoti G, Peddoju SK, Balasubramanian R (2014) Detection of Insider Attacks in Cloud based e-Healthcare Environment. 2014 Int. Conf. on Information Technology (ICIT), Bhubaneshwar, India. pp: 195-200. 
55. Nkosi MT, Mekuria F (2010) Cloud Computing for Enhanced Mobile Health Applications. 2010 IEEE 2nd Int Conf on Cloud Computing Technology and Science (CloudCom), Indianapolis, USA. pp: 629-633.

56. Yu WD, Kollipara M, Penmetsa R, Elliadka S (2013) A Distributed Storage Solution for Cloud Based e-Healthcare Information System. 2013 IEEE 15th International Conference on e-Health Networking, Applications and Services (Healthcom), Lisbon, Portugal. pp: 476-480.

57. Zhou J, Cao Z (2015) PPDM: Privacy-preserving Protocol for Dynamic Medical Text Mining and Image Feature Extraction from Secure Data Aggregation in Cloud-assisted e-Healthcare Systems. IEEE Journal of Selected Topics in Signal Processing.

58. Shih H, Chiang HS, Lin B, Lin SB (2010) An Embedded Mobile ECG Reasoning System for Elderly Patients. IEEE Transactions on Information Technology in Biomedicine 14: 854-865.

59. Boyle J, Bidargaddi N, Sarela A, Karunanithi M (2010) Automatic Detection of Respiration Rate From Ambulatory Single-Lead ECG. IEEE Transactions on Information Technology in Biomedicine 13: 890-896.

60. Lin CH (2010) An Intelligent Telecardiology System Using a Wearable and Wireless ECG to Detect Atrial Fibrillation. IEEE Transactions on Information Technology in Biomedicine 14: 726-733.

61. Bianchi AM, Mendez MO (2010) Automatic Detection of sleep macrostructure based on a sensorized T-shirt. 32nd Annual Int Conf of the IEEE EMBS (EMBC 2010), Buenos Aires, Argentina. pp: 3606-3609.

62. Yoo HJ, Yoo J, Yan L (2010) Wireless Fabric Patch Sensors for Wearable Healthcare. 32nd Annual Int Conf of the IEEE EMBS (EMBC 2010), Buenos Aires, Argentina. pp: 5254-5257.

63. Cheng $Y$, Chen H (2013) Low-Power SoC Design and System Implementation for Medical Applications. 2013 First International Symposium on Future Information and Communication Technologies for Ubiquitous HealthCare (UbiHealthTech), Jinhua, China. pp: 1-5.

64. Izumi S (2014) A 6.14 A Normally-Off ECG-SoC with Noise Tolerant Heart Rate Extractor for Wearable Healthcare Systems. 2014 IEEE Biomedical Circuits and Systems Conference (BioCAS) Lausanne, Switzerland. pp: 280-283.

65. Wang CP, Lee SY, Lai WC (2013) An RFID Tag System-on- Chip with Wireless ECG Monitoring for Intelligent Healthcare Systems. 35th Annual Int. Conf. of the IEEE EMBS (EMBC 2013), Osaka, Japan. pp: 5489-5492.

66. Pani D (2014) A Device for Local or Remote Monitoring of Hand Rehabilitation Sessions for Rheumatic Patients. IEEE Journal of Translational Engineering in Health and Medicine 2: 1-11.

67. Sawand A, Djahel S, Zhang Z, Nait-Abdessela F (2014) Multidisciplinary Approaches to Achieving Efficient and
Trustworthy eHealth Monitoring Systems. 2014 IEEE/CIC International Conference on Communications in China (ICCC), Shanghai. pp: 187-192.

68. Hovakeemiana Y, Naik K, Nayak A (2011) A Survey on Dependability in Body Area Networks. 2011 5th Int. Symp. on Medical Information \& Communication Technology (ISMICT), Montreux, Switzerland. pp: 10-14.

69. Kumar $P$, Porambage $P$, Ylianttila $M$, Gurtov $A$, Lee $H J$, Lee $H J$, et al. (2014) Addressing a Secure Session-Key Scheme for Mobility Supported e-Healthcare Systems. 2014 16th International Conference on Advanced Communication Technology (ICACT), Pyeongchang, Korea. pp: 538-540.

70. Li M, Lou W, Ren K (2010) Data Security and Privacy in Wireless Body Area Networks. IEEE Wireless Communication. pp: 51-58.

71. Lim S, Oh TW, Choi YD (2010) Tamil Lakshman, Security Issues on Wireless Body Area Network for Remote Healthcare Monitoring. 2010 IEEE International Conference on Sensor Networks, Ubiquitous, and Trustworthy Computing (SUTC), Newport beach. pp: 327-332.

72. He D, Zeadally S (2015) Authentication Protocol for an Ambient Assisted Living System. IEEE Communications Magazine 53: 71-77.

73. Lee S, Kim H, Lee SW (2013) Security Concerns of Identity Authentication and Context Privacy Preservation in uHealthcare System. 2013 14th ACIS International Conference on Software Engineering, Artificial Intelligence, Networking and Parallel/ Distributed Computing (SNPD), Honolulu, USA. pp: 107-112.

74. Dhavan AP (2015) Current and Future Challenges in Point-ofCare Technologies: A Paradigm-Shift in Affordable Global Healthcare with Personalized and Preventive Medicine. IEEE Journal of Translational Engineering in Health and Medicine.

75. Hiremath S, Yang G, Mankodiya K (2014) Wearable Internet of Things : Concept, Architectural Components and Promises for Person-Centered Healthcare. 2014 EAI 4th International Conference on Wireless Mobile Communication and Healthcare (Mobihealth), Athens, Greece. pp: 304-307.

76. Zhang $Y$, Sun L, Song $H$, Cao X (2014) Ubiquitous WSN for Healthcare: Recent Advances and Future Prospects. IEEE Internet of Things Journal 1: 311-318.

77. Catarinucci L (2015) An IoT-Aware Architecture for Smart Healthcare Systems. IEEE Internet of Things Journal.

78. Velikova M, Lucas PJF, Heijden MV (2015) Intelligent Disease Self-Management with Mobile Technology. IEEE Computer 48: 32-39. 